See Article page 275 .

\section{Commentary: Sometimes it is a zebra}

\author{
Kristen Errico, MD, and Edward Y. Sako, MD, PhD
}

El-Dalati and colleagues ${ }^{2}$ present a case report of a patient who underwent a mitral valve repair for presumptive culture negative endocarditis. Postoperatively, she developed thrombocytopenia, deep venous thrombosis, and multiple arterial thrombi. Testing was positive for antiphospholipid antibodies. Despite extensive, multidisciplinary evaluation and treatment, she developed multiple strokes and bowel ischemia, and died as a result of multisystem organ failure, a scenario termed catastrophic antiphospholipid syndrome (APS). ${ }^{1}$ In the setting of such a rare entity, how can we prevent a similar outcome in our own patients? The best defense is education and a routine high index of suspicion.

As is sometimes seen in such cases, there were other, more common explanations for the clinical data at hand that turned out to be misleading. Given her prior deep venous thrombosis and pulmonary embolism, APS might have been considered in this patient, but her prothrombotic state could be explained by her history of endometrial cancer. The patient had negative blood cultures and the cultures of the excised vegetations yielded no growth. This was attributed to the antibiotics taken for several weeks before presentation. Common things are common, but as the authors did, sometimes we need to consider an uncommon explanation. Although the patient was tested and discovered

From the Department of Cardiothoracic Surgery, University of Texas Health Science Center at San Antonio, San Antonio, Tex.

Disclosures: Dr Sako has a financial relationship with Medtronic, Inc. Dr Errico reported no conflicts of interest.

The Journal policy requires editors and reviewers to disclose conflicts of interest and to decline handling or reviewing manuscripts for which they may have a conflict of interest. The editors and reviewers of this article have no conflicts of interest.

Received for publication Oct 1, 2021; revisions received Oct 1, 2021; accepted for publication Oct 13, 2021; available ahead of print Oct 19, 2021.

Address for reprints: Edward Y. Sako, MD, PhD, Department of Cardiothoracic Surgery, University of Texas Health Science Center at San Antonio, Mail Code 7841, 7703 Floyd Curl Dr, San Antonio, TX 78229-3900 (E-mail: sako@uthscsa.edu). JTCVS Techniques 2021;10:280-1

2666-2507

Copyright (C) 2021 The Author(s). Published by Elsevier Inc. on behalf of The American Association for Thoracic Surgery. This is an open access article under the CC BY-NC-ND license (http://creativecommons.org/licenses/by-nc-nd/4.0/).

https://doi.org/10.1016/j.xjtc.2021.10.025

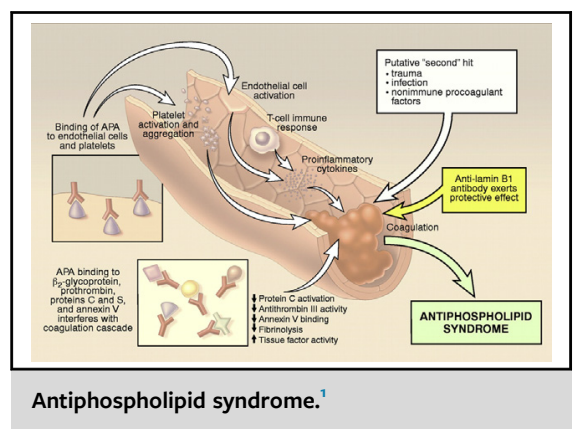

CENTRAL MESSAGE

Periodic review of rare periop-

erative conditions that have

catastrophic results is always

useful.

have antiphospholipid antibodies on postoperative day 4 , earlier diagnosis might have improved chances for a better outcome.

We agree with the authors that testing for APS should be considered in all patients with culture-negative endocarditis. We also suggest keeping APS on the list of differentials when a patient with microangiopathic hemolytic anemia is encountered. We test for heparin-induced thrombocytopenia not infrequently. As a method of maintaining a high index of suspicion, we should augment our list of differentials in these patients with other, less common causes, including APS, thrombotic thrombocytopenic purpura, hemolytic uremia, and drug-related microangiopathic syndromes. ${ }^{3}$

Finally, it should also be acknowledged that despite these efforts, laboratory testing is not perfect. In this case, the patient previously underwent testing for APS by lupus anticoagulant, but tested negative at that time. In addition, the mere presence of antiphospholipid antibodies does not confirm the diagnosis; however, it is possible to truly have APS with low levels of antibody titers due to consumption related to the thrombotic cascade. ${ }^{4,5}$ Furthermore, despite proper treatment with anticoagulation, corticosteroids, plasma exchange, intravenous immunoglobulins, and possibly cyclophosphamide, rituximab, and eculizumab, mortality is still $30 \%{ }^{3}$

Catastrophic APS will certainly not be the most common reason your patient has culture-negative endocarditis or postoperative thrombotic events, but as demonstrated, it can be. We thank the authors for presenting this case 
because it brings such a rare entity to light and demonstrates the devastating outcome that perhaps can be mitigated with recognition and a persistent high index of suspicion.

\section{References}

1. Hanly JG. Antiphospholipid syndrome: an overview. Can Med Assoc J. 2003; 168: 1675-82.
2. El-Dalati S, Suffoletto M, Rivosecchi RM, Kaczorowski D. Catastrophic antiphospholipid syndrome in the setting of mitral valve repair. J Thorac Cardiovasc Surg Tech. 2021;10:275-7.

3. Cervera R, Rodríguez-Pintó I, Espinosa G. The diagnosis and clinical management of the catastrophic antiphospholipid syndrome: a comprehensive review. $J$ Autoimmun. 2018:92:1-11.

4. Tebo A. Laboratory evaluation of antiphospholipid syndrome: an update on autoantibody testing. Clin Lab Med. 2019;39:553-65.

5. Drenkard C, Sánchez-Guerrero J, Alarcón-Segovia D. Fall in antiphospholipid antibody at time of thromboocclusive episodes in systemic lupus erythematosus. J Rheumatol. 1989;16:614-7. 